\title{
Applications of Internet of Things and Unmanned Aerial Vehicle in Smart Agriculture: A Review
}

\author{
Caprio Mistry $^{1}$, Ahona Ghosh ${ }^{2}$, Mousumi Biswas ${ }^{3}$, Bikalpa Bagui ${ }^{4}$, Arighna Basak ${ }^{5}$ \\ ${ }^{1}$ Department of Electronics and Communication Engineering, Brainware University, India \\ caprioc1p1@gmail.com \\ ${ }^{2}$ Department of Computer Science and Engineering, Maulana Abul Kalam Azad University of \\ Technology, West Bengal, India-700064 \\ ahonaghosh95@gmail.com \\ ${ }^{3}$ Department of Computer Science and Engineering, Brainware University, India \\ moubiswas2099@gmail.com \\ ${ }^{4}$ Department of Computer Science and Engineering, University of Engineering and Management, India \\ bikalpabagui13@gmail.com \\ ${ }^{5}$ Department of Electronics and Communication Engineering, Brainware University, India \\ arighnabsk@gmail.com
}

\begin{abstract}
With the rapid advancement of technology and decline in human ability, technology has become a part of every aspect of our lives. Agriculture and irrigation are two domains in which man's potential may be exploited to its fullest. To commercialise in the industry, a variety of sensors and electronics devices are employed to keep prices down in a few domains. In order to save money and enhance the abilities of agricultural experts, UAVs (unmanned aerial vehicles) can be used for reconnaissance, pesticide and insecticide application, and bioprocessing mistake detection. When it comes to this application, both single-mode and multi-mode UAV systems will work just fine. On the other hand, this chapter identifies the challenges and limitations of IoT and UAVs connection in remote locations, demonstrating several use cases of smart agriculture and the advantages and applications of using IoT and UAVs in agriculture.
\end{abstract}

\section{X1. Background}

An increase in the population has enormously augmented the burden on the agriculture sector. With the beginning of technology, this era is observing a shift from conventional methods to the most innovative ones. Regardless of the perception people may have about the agricultural development, the truth is that today's agriculture industry is data-cantered, accurate, and smarter than ever. The quick development of the Internet-of-Things (IoT) based technologies reformed almost every industry including "smart agriculture" which transformed the industry from statistical to quantitative approaches. Such radical changes are trembling the present agriculture approaches and generating new chances along a variety of challenges. Researchers, research institutions, academicians, and most nations across the globe are poignant towards the preparation and implementation of collaborative projects to explore the horizon of this field for helping manhood. The tech industry is competing to afford more optimum solutions. Inclusion of IoT, along with cloud computing, big data analytics, and wireless sensor networks can offer sufficient possibility to predict, process, and examine the circumstances and recover the activities in the real-time scenario. The perception of heterogeneity and interoperability of the devices by providing flexible, ascendable, and strong methods models are also maiden new areas in this field.

IoT can be used in every mode of life: smart cities, smart homes, smart retail and many more. Using IoT in agriculture repetitions is the essential of the period, as the global population will knockout a peak of 9.6 billion by 2050, to chance that kind of demand the agriculture industry desires to supply at an even faster rate. This is completed by using 
modern technology and mainly IoT. IoT makes labour free farms a possibility. In not only major farming practices but also it can also be used in maintaining livestock, greenhouse farming, managing farms etc. The most significant apparatus used for IoT is Sensors. Sensors are devices that collect important data, which is taken to get the anticipated analysis. For agriculture, sensors are mainly used to acquire readings, determine NPK values, and detect diseases \& moisture content in the soil. Study nad investigation of IOT discovers its application in the agricultural sectors. Smart agriculture is called precision agriculture as it practices exact data to reach conclusions. It displays the various sensors, which help IoT and agriculture, their applications, challenges, advantages and disadvantages.

Therefore, technology is being involved in every stage of our life with the speedy technological enhancement and decrease in human capacity. Agriculture and irrigation are two fields where man's ability can be realized. Various sensors and electronics devices are used to commercialize in the industry while keeping costs down in a few realms. UAVs (unmanned aerial vehicles) can be used for reconnaissance, pesticide and insecticide application, and bioprocessing error detection to save money and improve the skills of agricultural professionals. Both single-mode and multi-mode UAV systems are suitable for use in this application. The expertise of a single UAV system can be surpassed by the right cooperation and synchronization of a network of UAV clusters attached to ground infrastructures, GCS or satellites. Therefore, the major contribution is that the mobility model and specifications are the most effective routing protocol for each specific agricultural application.

\section{X2. Introduction}

The word 'Agriculture' is contingent from the Latin words 'Ager' means 'Land' and 'Culture' means 'Cultivation'. It is the landmark of human development and is one of the benchmark areas. To progress the agricultural production with fewer properties and efforts, extensive revolutions have been prepared through human history. Yet, the high population level never let the demand and supply match throughout all these times. More than $60 \%$ of the human population depends on agriculture for existence and around $12 \%$ of the total land area is under agricultural production as per consideration of the Food and Agricultural Organization of the United Nations (FAO) [1]. According to the forecasted scenario, in 2050, the world population is predictable to touch 9.8 billion, an upturn of approximately $25 \%$ from the current situation [2]. Almost the whole declared rise of population is predicted to occur among the emergent countries [3]. Moreover, on the other side, the trend of development in urban areas is predicted to stay at an accelerated pace, with about $70 \%$ of the world's populations forecast to be built-up until 2050 [4]. In India, approx. 50\% of the population is dependent on the agriculture region for occupation and approx... $61.5 \%$ of the Indian population is predominantly dependent on the agriculture region for its livelihood [5-6].

However, throughout the last few decades, agriculture is experiencing a fourth revolution by assimilating Information and Communications Technologies (ICT) in traditional farming [7]. Technologies, like Big Data Analytics (BDA) and Machine Learning (ML), Remote Sensing, Internet of Things (IoT), Unmanned Aerial Vehicles (UAVs) are mostly auspicious and they can bounce an innovation in agricultural systems [8-9]. In smart farming, a extensive range of agricultural constraints can be observed to expand crop yields, to ease costs and improve process inputs, such as environmental circumstances, development status, soil status, irrigation water, pest and fertilizers, weed management, and greenhouse production environment [10]. Smart farming is a green technology methodology, since it decreases the environmental footmark of traditional farming [9]. In precision agriculture, smart irrigation and the negligible usage of fertilizers and insecticides in agricultural crops 
can further diminish leaching difficulties and productions, as well as the impact of climate change [9], [11]. IoT is one of the most radical technologies in contemporary wireless communications [12]. The basic idea is the communication between a variety of physical belongings or objects using specific addressing patterns to being connected to the Internet. IoT technology can be realistic in various vertical markets including transportation, healthcare, industry, vehicles, smart home, and agriculture [13]. In an agricultural system, IoT devices offer useful information on a wide range of physical constraints to improve cultivation process [10]. The part of Wireless Sensor Networks (WSNs) in IoT technology is of ultimate reputation since the huge majority of IoT applications in numerous marketplaces is based on wireless data transmission.

The ubiquitous use of the Internet in the last two decades has provided organisations and people around the world with unrestricted benefits. The main advantage of this breakthrough was the potential to provide real-time production and customer services. The Internet of Things (IoT) has recently promised to provide the same benefits with its revolutionary innovations and to increase customer awareness and capabilities by changing the operating environment. In the areas of health, shopping, traffic, Défense, intelligent homes, Smart Cities and agriculture, IoT offers a number of solutions. In agriculture, IoT implementation is seen as the optimal solution, since continuous monitoring and control is essential in this sector. IoT is seen on a variety of occasions within the manufacturing chain of agriculture [14]. The framework built is even more interesting in dealing with node faults and rearranging the weak connectivity ties on the network on their own. In [15] an IoT management is proposed that tracks wind, soil, atmosphere and water elements across a wide range of areas. In addition, IoT-based solutions for agricultural surveillance have been established based on their subdomains. Sub-domains listed include soil tracking, air surveillance, temperature monitoring, water monitoring, disease monitoring, location surveillance, environmental monitoring, insect monitoring, and fertilization surveillance [1617]. Precision farming, livestock and greenhouses, which are clustered under various surveillance areas, are the major applications of IoT in agriculture. The wireless sensor network (WSN), which helps farmers collect relevant data via detectors, is used to track all these applications with different IoT-based sensors/devices. Certain IoT-based settings analyse and process remote data through cloud providers, helping scientists and farmers to decide better. Today, environmental control systems provide additional management and decision-making facilities through the development of current technologies. A custom landslide Risk Management System has been designed to enable fast deployments without user interference in hostile environments [18]. Data from various environmental parameters are furthermore conveyed to the user by warnings or by message to the authorities [19].

The aim of smart agriculture is to increase production, yields and profitability and minimize the environmental performance through the use of various methods such as effective irrigation, targeted and accurate use of pesticides and fertilizers on crops, etc. The evolving internet of objects (IoT) and unmanned aerial vehicles (UAVs) have made sustainable smart farming possible. IoT brings value to its data by automated collection, interpretation and access as a major technology for intelligent agriculture by ensuring that data flows between various devices such as sensors, relays and gateways. This makes production and management processes in smart farms more cost-effective and timely [20].

In addition, IoT decreases the effects of inherent environment by allowing for reactions in real time to infestations such as weeds, pest or disease identification, weather tracking and prevention, soil conditions etc. As a result, UAV and IoT technology make it easier to adequately exploit commodities like water, nutrients or agro-chemicals. Moreover, 
the qualities of crop yield and environmental impacts from agriculture have been improved with these intelligent technologies. Since some of the main characteristics of IoT and UAVbased smart farming are [21-23]:

a. Field monitoring: Smart agriculture aims to reduce crop waste by improved surveillance, precise data collection and data analysis.

b. Smart farming aims to detect the place of animals that graze in open areas in large stables. Views and tracking: Technology also helps assess the air quality and ventilation condition of farms and identifies toxic excrement gasses.

c. Greenhouse applications: Smart agriculture monitors micro-climate conditions with the goal of improving fruit and vegetables production and quality in green houses.

d. Biomass management: Smart farming helps to regulate humidity and temperature in crops like straw, grass, etc. as a measure for avoiding fungus and other microbial pollutants.

e. Biomass management: Smart farming helps to regulate humidity and temperature in crops like straw, grass, etc. as a measure for avoiding fungus and other microbial pollutants.

f. Offspring Care: Intelligent breeding regulates the raising conditions and wellbeing of offspring in animal farms.

Moreover, the applications of UAVs are varied, including areas related to inhabitant, military, commercial, and governmental sectors [13-18]. Examples contain environmental monitoring (e.g., pollution, health of plants, and industrial accidents) in the civilian sector. In military and governmental zones, the UAVs are used for surveillance and delivery applications pointing to obtain information at locations after a disaster or outbreak, and to distribute medicine or other essential items. Commercial applications are absorbed on transporting products and goods both in urban and rural areas. UAVs, subsequently they are dependent on sensors, antennas, and embedded software, are considered as part of the IoT, obtaining a two-way communication for applications associated to remote control and monitoring [19]. The IoT creates a quickly emergent cutting-edge environment in which the principal concept lies in the orchestration of a large range of smart objects in such a way that these can be exploited and activated globally, either directly by users or by special software that captures behaviour and ideas. IoT allows objects to develop active participants of everyday activities, with abundant promising applications through various communication technologies in the background of the "smart-city" vision [20]. It is projected that around 30 billion uniquely recognizable objects are expected to be part of this global community by 2022. These forecasts are expected to significantly rise with the introduction of $5 \mathrm{G}$ technologies with UAVs.

The goal of UAVs, on the other hand, is to provide more perspectives such as imaging analysis and agricultural surveillance in intelligent farming [24]. UAVs not only promote image analysis and agricultural field processing, but also enable a thorough awareness of the situation by patrolling a field of interest [25]. In addition, UAVs can be used to provide the grounded tracking stations with valuable information through data transmission. In several agricultural areas the uses of UAV are extended, including the prospecting and spraying of insecticides and fertilizers, seed planting, weakening identification, fertility evaluation, mapping and planting. These recent advances in intelligent IoT and UAV-based agriculture help the world achieve the '2030 Sustainable Development Agenda' priorities, where United Nations (UN) and international communities are setting the goal of eliminating hunger by $2030[26]$.

\section{X.3. Unmanned Aerial Vehicle (UAV)}


Unmanned Aerial Vehicle is a low-cost substitute element in sensing technology and data analysis method in the latest years. Fundamentally, remote sensing by electromagnetic energy measures the belongings of targeted object from a distance and has the advantages of comprehensiveness, non-invasiveness, timeliness, and flexibility. However, remote sensing determine soil properties in the farmhouse is far away from actual data for the complex natures of remote sensing, agricultural production and soils [27-29]. According to researchers, UAV can be a choice to gather accurate data in the field. It had been recognized as a possible technology that can produce high spatial resolution imagery $(<1 \mathrm{~m})$ and at temporal frequency suitable for timely responses in the creation of actionable information about crop and field status [30]. The small UAV fits to Low Altitude Remote Sensing (LARS), which is less as expensive compared to conventionally manned aircraft is possibly one of the main reason why the UAV production had been exceeding the market demand [31].

Although, an Unmanned Aerial Vehicle (UAV) is a kind of aircraft that works without a human pilot on-board. There are dissimilar types of UAVs engaged for different purposes. Originally, the technology was engaged by the military for anti-aircraft target practice, intelligence congregation and surveillance of some enemy territories. The technology has though grown beyond its primary purpose and in recent years has increased importance in different spheres of human effort. A progression in technology has permitted for the augmented adaptation of unmanned aerial vehicles for several purposes. Without an on-board pilot, UAVs are organized either remotely by a pilot at a ground station or separately, steered by a pre-programmed flight plan. There is a enormous potential for the application of UAVs in Agriculture [32]. One such application is in accurate and evidencebased forecasting of farm produce using spatial data collected by the UAV. UAVs also permit farmers to detect their fields from the sky. This sky-view can expose many problems on the farm, common among which is irrigation related problems, soil variations, fungal and pest infestations. Moreover, information connecting to water access, varying climate, wind, soil feature, the occurrence of weeds and insects, flexible growing seasons, and more can all be observed with UAVs. From a livestock perspective, UAVs are being used to accomplish head counts, observing animals and reviewing eating habits and health related patterns. Utilizing the information gathered, farmers can provide fast and efficient solutions for detecting problems and issues, make better administration decisions, recover farm productivity, and ultimately produce higher revenue [33-34].

The advantages of UAV are, it can detention the pictures of the farmer's crop with a variation of camera filters that can offer the farmers with multiple spectral imaging, sanction the image processing and investigation, which gives better information regarding their crop's health and at the same time recognising areas of the crops that involve specific forms of attention. The small UAV can be easily hovered and preserved with little training, production it a great choice for farmers watching to development their farming by merging agriculture by the knowledge of remote sensing [33-35].

\section{X.4. Types of UAV}

Multiple principles can be used to achieve an unmanned aerial vehicles classification [8], such as size, Maximum Take-off Weight (MTOW), range etc. Moreover, these will be considered where UAV flight regulation is examined. The classification has been prepared with the wing type and autonomy level that could be the most appropriate standards in agricultural missions [36-39]. 
According to the category of wing, two main sets can be observed: rotary and fix wings. The first group is prepared up of helicopters and multi-rotor (usually known as drones). The airflow is formed by numerous rotors that produce the suitable trust for lifting. Their main advantage is the capacity to achieve hovering flights, which is applicable to aerial photography since it permits increasing the capture time for the cameras and therefore reimburse poor light situations. Moreover, the airflow demonstrations great performance at low speeds and agree low altitude flights with minimum risk. Multi-rotors have converted to more popular owing to their mechanical ease in contrast to helicopters, which rely on a much more refined plate control mechanism. Therefore, the multi-rotor flight is observed just by changeable the velocity of several direct current (DC) motors, without any mechanical moving machinery, in difference to helicopters. Moreover, the reduction in price of brushless motors, which need no maintenance, as well as their electronic supervisors, has made them an inexpensive resolution for multiple responsibilities in civil applications. Owing to this, a significant number of drone producers have appeared in the market for providing a wide range of systems. The main inadequacy of commercial drones is their inferior payload capability in comparison with helicopters. But, drone manufacturers have augmented the number of rotors from 4 to 6 or 8 consequently as to decrease this gap and presently drones with a maximum payload proficiency of $22 \mathrm{~kg}$ [40-43] are presented on the market. Not only does payload capacity rise with the number of rotors, but there is similarly an development in safety, subsequently the aircraft typically is capable to fly in depreciate mode when one or even more rotors is under failure, permitting for arrival in a safe method. Alternatively, fixed wing aircraft, like planes, require generating airflow to boost by moving their aerodynamic surfaces (wings and ailerons) at high velocity. Consequently, the aircrafts are not able to execute static flights. Subsequently the velocity cannot be decreased in the same way as rotary wings, they usually involve higher altitude to achieve safe flights. Further, the manoeuvrability exposed by rotary wings (e.g., instant rotations on vertical axis) is a abundant advantage over those with fixed wings. The maximum range for a secure wing aircraft can stretch is higher than rotary wings, owing to legal guidelines for flying UAVs, it does not develop a real benefit in most of the circumstances, and even nevertheless for the most part, the aircrafts exhibit a advanced payload capacity. Therefore, the analysis agreed that rotary wings aircrafts are mostly favoured for agricultural responsibilities than those with fixed wings.

\section{X.5. IoT for smart agriculture}

Smart agriculture is a contemporary farming concept with IoT technology to rise the productivity in agriculture. Using smart farming, farmers can successfully use fertilizers and other resources to escalation the quality and quantity of their crops. Farmers cannot be contemporary on the field 24 hours a day. Moreover, the farmers could not have the information to usage different apparatuses to measure the ideal ecological conditions for their crops. IoT offers them with the automatic system, which can function deprived of any human direction and can inform them to make proper conclusion to deal with different kind of difficulties they might face during farming. It has the ability to spread and inform the farmer even if farmer is not on the ground, which can allow farmer to achieve more farmland, thus cultivating their production. it was estimated that the universal population will extent 9 billion mark by 2050 [1-5]. Therefore, IoT applications are must for farming to nourish such large population and efficiently use the farmland and other possessions, as they are scarcely available in some places. For Global warming, random weather situations is affecting the crops and farmers are facing losses thus the IoT Smart Farming application will allow them to take quick actions to avoid that from happening [44-51]. 
However, There are some significant constituents of Agricultural IoT (AIoT), such as sensor equipped devices, internet connectivity, wireless communication technology, sensed and transmitted data, etc. The wireless communication technology shows an essential role in the effective arrangement of IoT systems, which can be characterized based on transmission distance, spectrum, and application scenarios. As shown in Fig X.1, the construction of IoT is mainly based on three layers; namely, the Physical layer where the sensing is done, the network layer which contracts with data transfer, and the application layer where storage of data and data manipulation are observed [46].

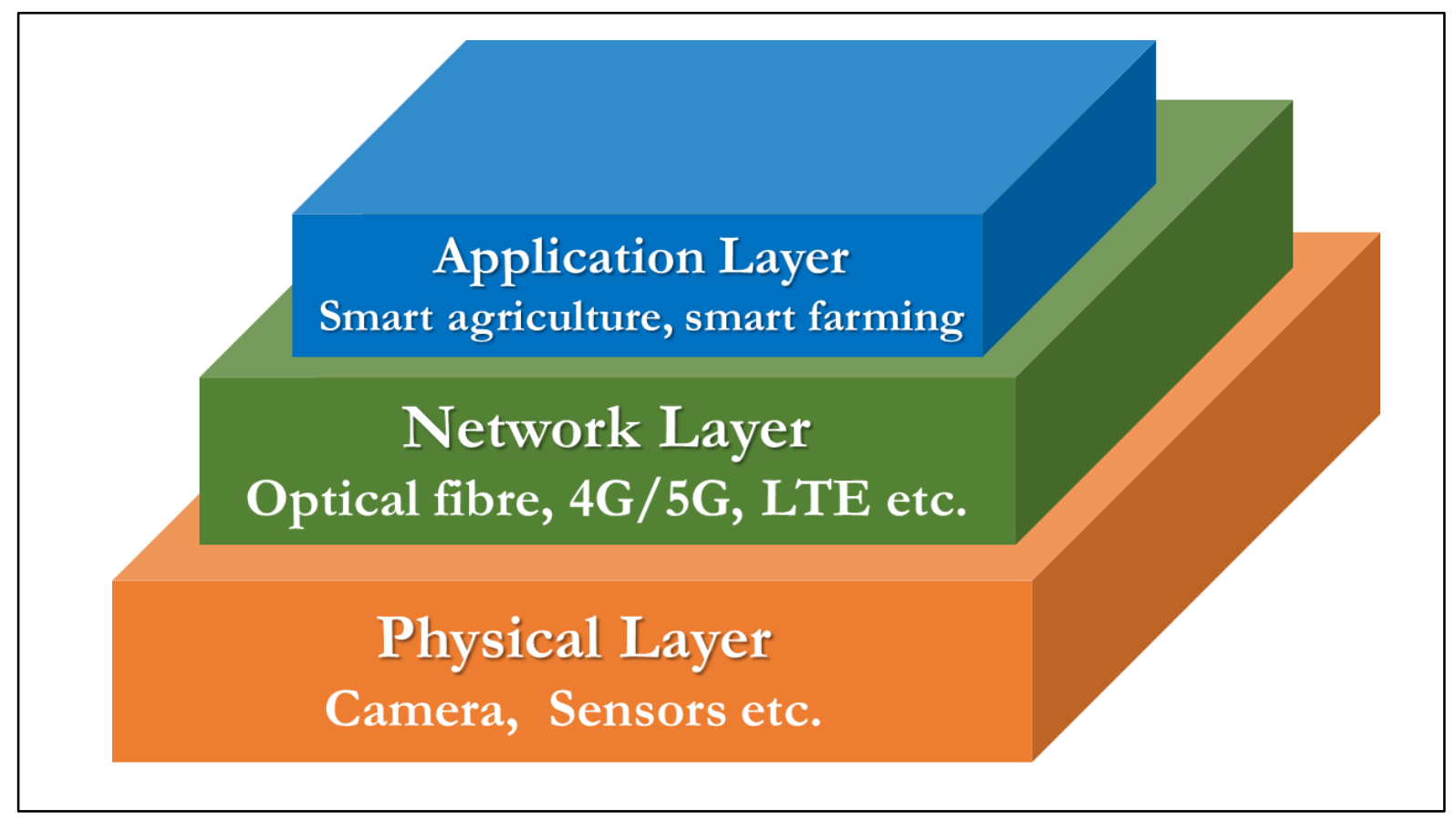

Fig X.1. IoT architecture

\section{X.5.1. Physical Layer}

The physical layer comprises of several terminal devices, camera, sensors, Wireless Sensor Networks (WSN), Radio Frequency Identification (RFID) labels and readers, Near Field Communications (NFC) devices, etc. [52]. For this layer, sensors are used to assemble information about wind speed, temperature, humidity, nutrient level, plant diseases, insect pests, etc. The composed information is being managed by the embedded devices and is uploaded to a higher layer for additional processing and investigation. This terminal devices and sensors are recycled to monitor, control, and recognize agricultural and livestock goods. For example, WSNs are regularly used for controlling and monitoring of storage and logistics services [52]. Further, on the other side, RFID technologies are the most vital pattern for interconnected devices. RFID tags are used to store data by Electronic Product Code (EPC), which are then read, activated and operated by RFID Readers. Therefore, by contribution the object identification, tracking, monitor, control, and data storage on active or passive devices; WSN, RFID and NFC technologies are used to play a significant role in the agricultural domain.

\section{X.5.2. Network Layer}

In the network layer, the sensors and devices are essential for connection with the adjacent nodes and a gateway in order to construct a network. At this layer, the sensor nodes cooperate and interconnect with other nodes and gateways within a network for forwarding data 
towards a remote structure; where the data are kept, further investigated, handled and dispersed for useful information [53].

\section{X.5.3. Application Layer}

The uppermost level of the IoT architecture is the application layer, where the assistances and advantages of IoT are outward. There are heaps of bright stages or systems in this layer to control and observe the soil condition, water and nutrition level, plants and animals. These layers also used to support the initial warning and organisation of diseases and insect pests, infestation and agricultural creation safety controllability; as an outcome, manufacturing efficiency can be enhanced

\section{X.6. Communication technologies used in IOT based smart agriculture}

Wireless protocols and standards fixed the procedure for wireless communication. For example, IEEE 802.15.4 is a wireless standard, which is used to allow the connection between internet-enabled gateways and end-nodes. Further, the other examples are ZigBee, Sigfox, EC-GSM, ONE-NET, Wireless HART, ISA100.11a, LoRaWAN, Bluetooth Low Energy (BLE), DASH7, etc. [54]. These standards are classified in terms of transmission ranges, which are shown in figure [55]:

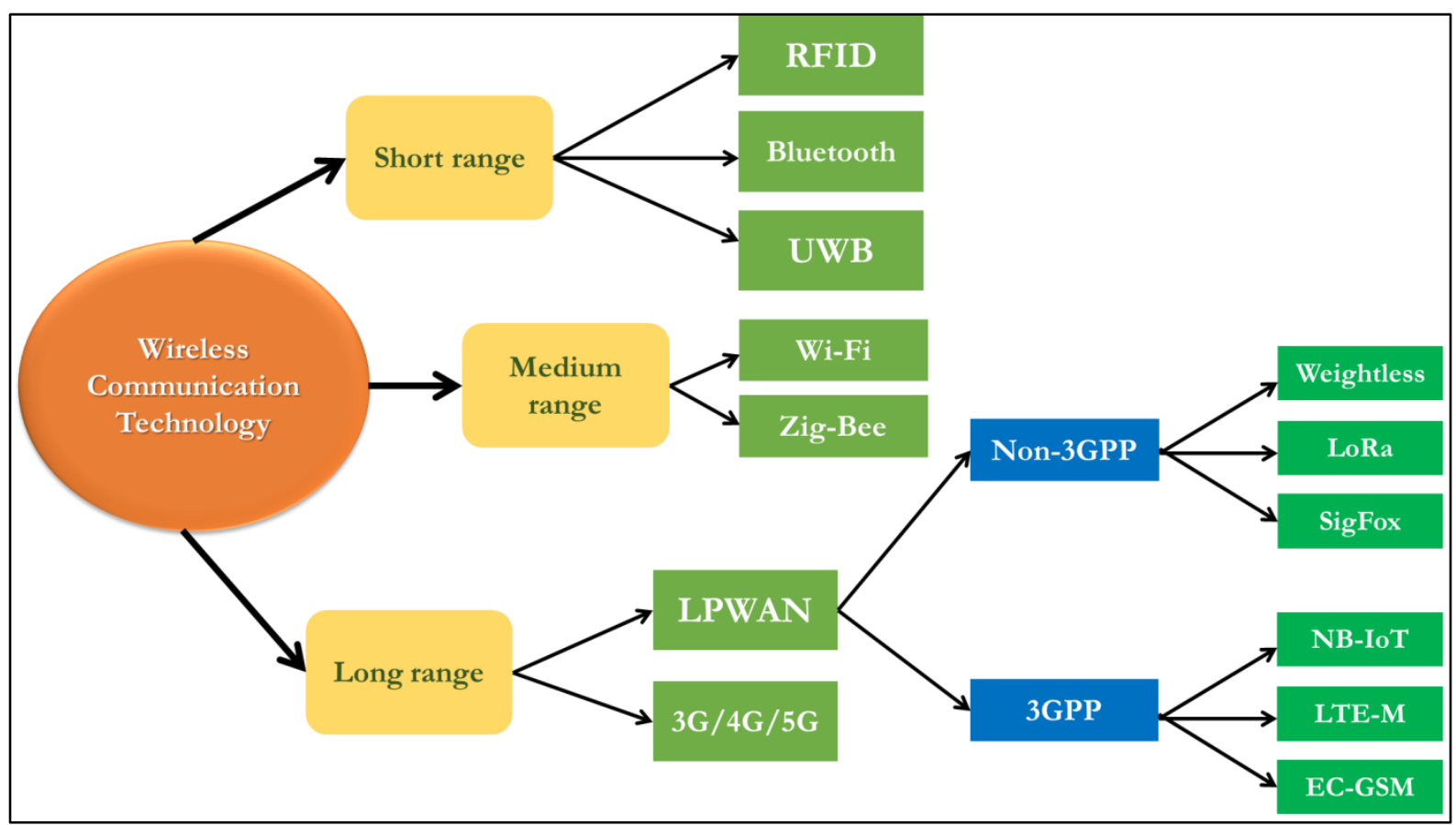

Fig. X. 2.: Classification of Communication Technologies for IOT based smart agriculture

For short-distance wireless communication applications, IEEE 802.15.4-based protocols are the ideal choice [53], which is projected for Low-Power Wide-Area Networks (LPWANs). It uses a data rate between $20 \mathrm{kbps}$ to $250 \mathrm{kbps}$, works on different frequency bands like 433 $\mathrm{MHz}, 868 \mathrm{MHz}, 915 \mathrm{MHZ}$ and $2.4 \mathrm{GHz}$; and helps a maximum outdoor Line of Sight (LoS) range of $100 \mathrm{~m}$ [56]. On the conflicting, IEEE 802.11 standard are appropriate for applications, which are essential for medium range connectivity. Further, IEEE $802.11 \mathrm{p}$ is another choice for incorporate situations with a high mobility condition. This specific issue seems to be of large attention in agriculture circumstances because of its large communication range, maximum legal transmission power of $1 \mathrm{~W}$, and the less interfered band of $5.9 \mathrm{GHz}$ ISM frequency [50]. When coverage is concerned, long- distance communication technologies are the only dependable and anticipated solutions. Cellular 
communication technologies such as 3G, 4G, LTE, and 5G are most appropriate and a dependable standard for precision agriculture, where a big quantity real-time data requirements for transmission and processing [57]. Further, the 5G communication technology is predictable for providing real-time Device to Device (D2D) communication, which will allow vehicle location. Moreover, a enormous amount of devices can be maintained per square kilometer [58]. In contrast to LTE, 5G can activate on advanced frequency bands, and therefore can work on wider channel bandwidths. Mainly in rural areas, $5 \mathrm{G}$ communication technology may allow new abilities on farm apparatus by contribution of higher data rates and larger transmission range under the model of real-time connectivity. However, the obtainability of the cellular network and the economic possibility of $5 \mathrm{G}$ technology in rural areas is a questionable distress.

IEEE 802.11ah and LoRa/ LoRaWAN are the most consistent among long distance communication technologies of IoT. Moreover, the first one is an alteration of the IEEE 802.11 family, which was available in 2017 to help IoT setups such as smart monitoring [50]. It uses $900 \mathrm{MHz}$ bands, and delivers wider coverage distance and uses lower energy as compared to IEEE 802.15.4. It delivers connectivity to thousands of strategies with a single access point up for a radius of one-kilometer. On the conflicting, LoRaWAN is another most auspicious LPWAN specifications projected for a system of battery-operated wireless nodes.

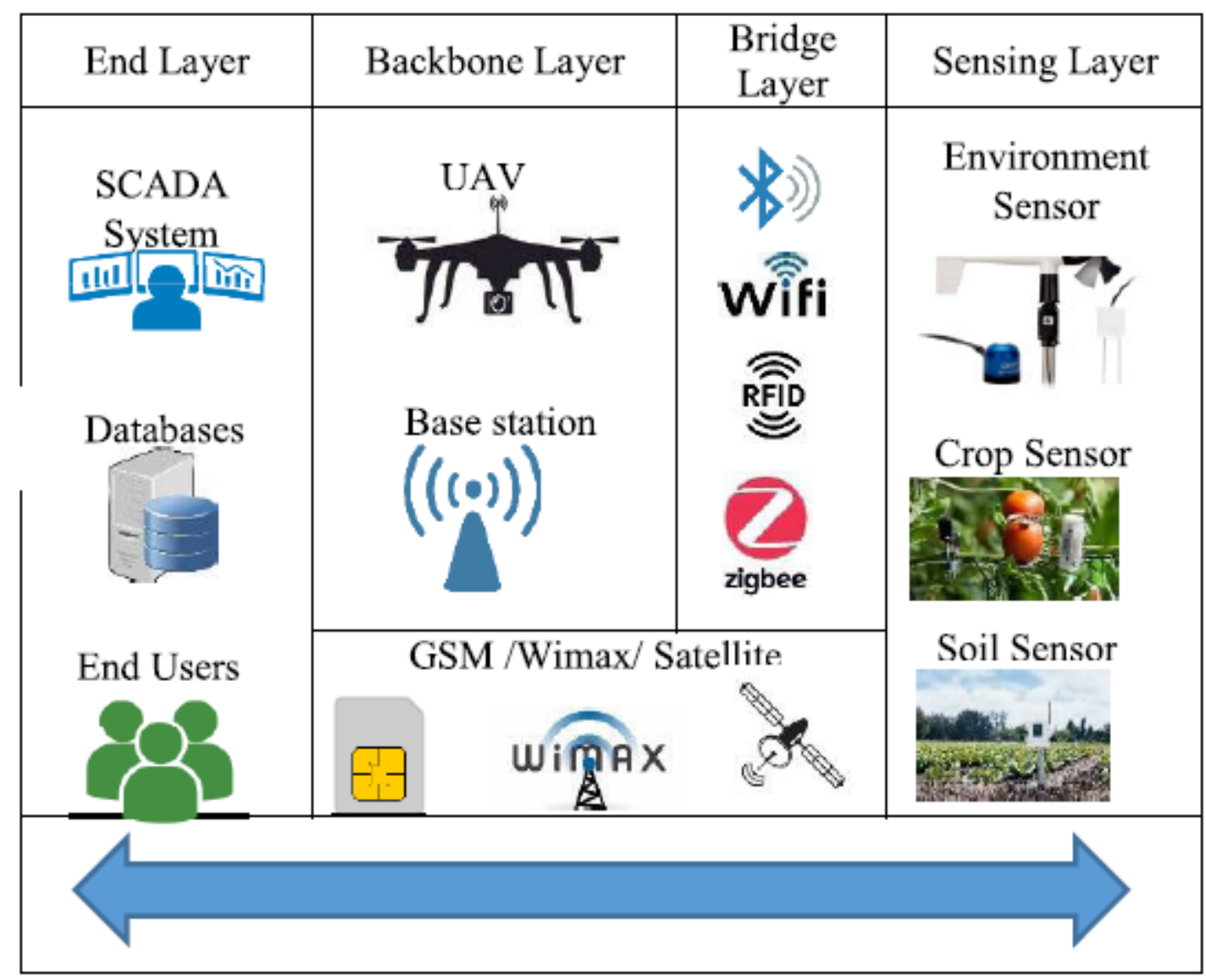

Fig X.3. End to end communication for smart farming [44]

Cellular communication approaches from $2 \mathrm{G}$ to $4 \mathrm{G}$ can be appropriate, dependent on the purpose and bandwidth necessity; however, the dependability, and even accessibility, of a cellular network in rural areas is a main apprehension. To overcome this, data transmission through satellite is another choice, but, here, the price of this communication method is very 
high, which creates it not appropriate for small- and medium-sized farms. The superior of communication mode also be contingent on application requirements, like some farms require sensors that can activate with low data rate but necessity to work for long periods therefore requires long battery life. For such situations, a new choice of Low Power Wide Area Network (LPWAN) is selected as a better resolution for cellular connectivity, not only in positions of long battery life but also in positions of a larger connectivity choice with reasonable rates [59]. Presently, crop and pasture management are the key applications where LPWAN networks are highly appropriate, where it can be exploited in many other farmingrelated usages.

Further, for farming UAVs, the Micro Air Vehicle Link (MAVLink) is a shared communication etiquette permitting UAVs to communicate with Ground Control Station (GCS). It interconnects between calculating platform, monitoring platform of UAV and applications platform of GCS [60]. MAVLink is used to transmit directions, location of global navigation satellite system (GNSS) and velocity of the UAV. The communication space between the UAV and the GCS depends on conditions of the UAV. Conversely, it can connect up to $2 \mathrm{~km}$ when the UAV is inside LOS [61]. Presently, UAVs are planned to work at return-to-launch mode, which allows the UAVs to return automatically to its first position when communication is disturbed. This method is triggered to stop unwanted accidents or loss of UAVs [47,62-63]. Few additional types of communication systems are also accessible for communicating between GCS and UAVs like ZigBee, radio-frequency modules, and other communicating devices. The communication range can be improved with use of additional technologies, including phone apps. Furthermore, the appearance of 5G cellular technology abilities to greatly recover infrastructures and data-processing speeds, which will be valuable for high-definition plotting [64-65].

\section{X.7. UAV for smart agriculture}

Now a day, the IoT system has prepared remarkable progress in many industries, comprising farming regions. but when taking on agriculture, the communication amenities like base stations or Wi-Fi are very inadequate, which inhibits the development of the IoT in this sector. Such communication arrangement and associated amenities are even worst in emerging counties and rural areas, which is one of the main steeplechases when presenting the IoT in the agriculture industry. The data developed by the wireless sensors cannot be communicated in the absence of reliable communication arrangement. In such a situation, UAVs make an alternate way for this purpose. The UAV system connects with the wireless sensors range over large extents in order to produce data for advance processing and investigation. One of the best valuable revolutions of smart agriculture are the robots, among which, UAV, also entitled drones, have been broadly applied [48, 66]. Drones or UAVs are being extensively used by agriculturalists for farm development observing and controlling. Some of UAVs are being cast-off to spray water, other insecticides powerfully in the rough lands were human movement is not easy, and the crops retain dissimilar heights.

Additionally, UAVs, better acknowledged as drones, fixed with high-resolution cameras and exact sensors, can be hovered over thousands of hectares of farms. The role of investigation in all agriculture applications is extremely serious, particularly in forestry and crop observing for necessary to cover large zones [67]. Consequently, a fast, low-cost, actual, and extensive surveillance maintained with an exact data attainment and broadcast facility is vital for agriculture manufacture. Presently, mostly two choices are used to find aerial images of a field or farm zone: satellite and airplanes. Both of the choices are decent for a macro view of scenery, but the choices face severe problems related to their quality when it comes to PC views. These images are not decent in resolution and cannot provide quality of the 
image, which is mandatory throughout the investigation. Furthermore, the visiting frequency also face problems and it is not easy to collect images frequently. Moreover, another severe problem is that these control beyond the cloud level where there is a solid probability that both are congested in bad climate.

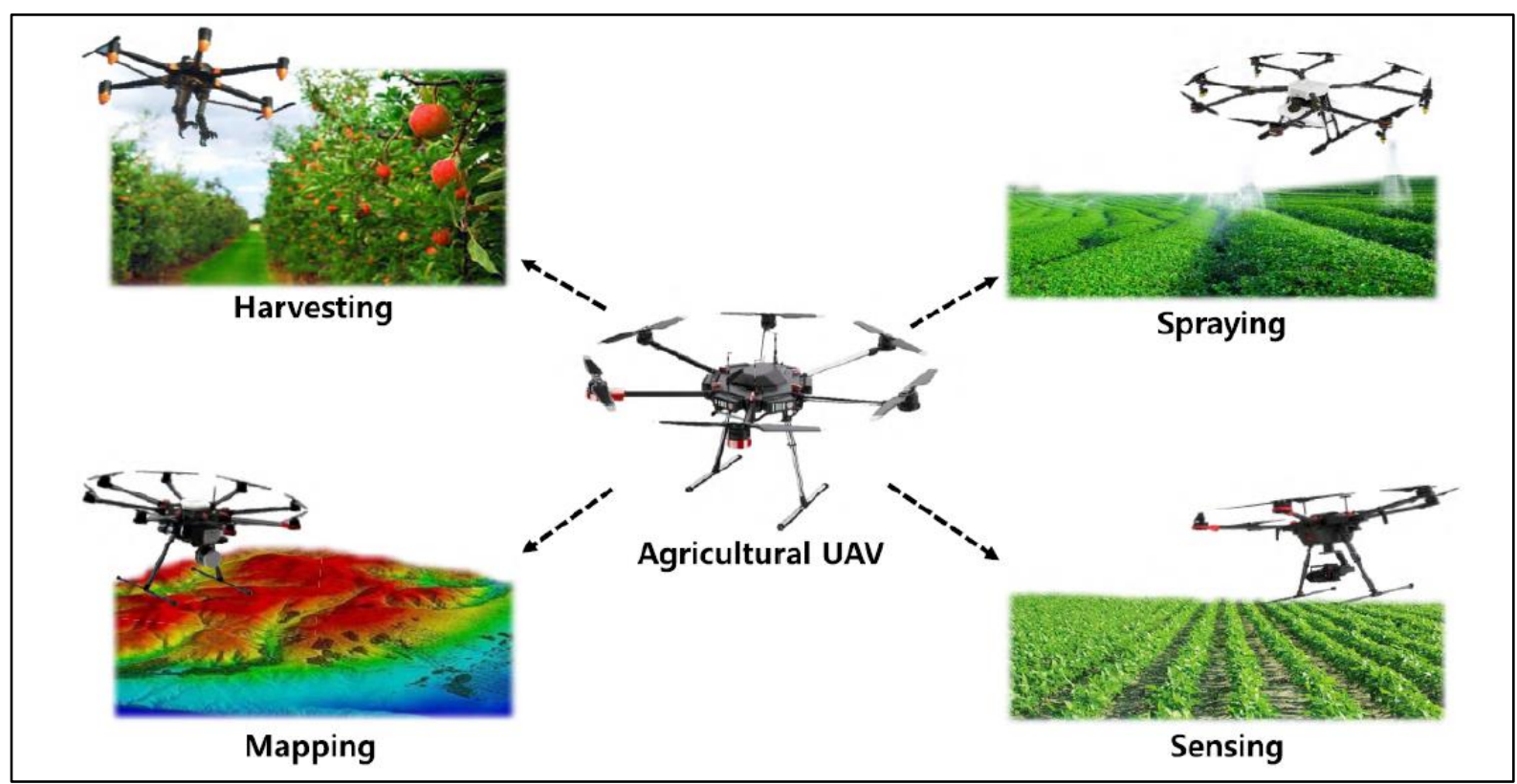

Fig X.4. Different types of agricultural UAVs [47]

However, UAVs is a platform which provides an "eye in sky", that overcome or remove the above stated problems related to the micro views. The value of images occupied through UAVs depends on the involved cameras resolution usually many times improved than satellite pictures and, most significantly, regulates according to application desires. More specifically, UAVs maintenances faster and better NDVI to assess crop circumstances like weed mapping, leaf assessments, etc., and offer instant response to the farmers for timely movements. Correspondingly, UAVs are enhanced in terms of frequency, even if desires multiple times in a single day, and affects by weather conditions, unless it is raining. Owing to the stated advantages, UAVs are chosen as the future of precision agriculture.

Not only incalculability, but in certain applications of insecticide and fertilizer, UAVs requirement to transport heavy payloads. Therefore, in such situations, optimum battery consumption becomes vital to spread the flight time. For this resolution, many parameters can be measured to enhance the drone efficiency. Initially, when flying, select right conditions e.g. climate or air direction. Succeeding, attempt to comprise optimum payload and place it properly. For this condition, it can be supportive to attribute the payload near the field, improved in smaller numbers and refilling again in its place for putting heavy quantities. Moreover, depending on area size and visiting frequency, optimum path choice shows a serious role. For this resolution, many routing arrangements like [259], [260] are projected particularly for the UAVs so selecting and realizing the right arrangement can deliver clear modification. As the application of pesticide and UAV based agriculture where drone essential to fly with weighty payloads then new actions like tethering system can be supportive. In UAV tethering, a assembly that offer power through the long cable, is delivered so that it can wing as long as the farmers have power backup on the field, most significantly it doesn't need to lift heavy batteries. Presently, agriculture is actuality considered one of the most approving fields where UAVs can propose many solutions to resolution many leading and long-lasting problems. Some of important applications in which 
drones are already playing important roles to support farmers through the system model are highlighted below.

\section{X.8. Applications of IoT and UAV in Smart Agriculture through system model}

IoT and UAVs can be employed in many application areas of smart agriculture. This section deliberates some applications of IOT and UAV in smart agriculture through system model.

\section{Smart crop monitoring}

Crop monitoring states the appropriate sensing of different constraints of a farm. Automatic observing is one of the introductory features of smart agriculture. Advantageously positioned sensors can spontaneously sense and convey data to a gateway for supplementary investigation and controlling. Sensors are used to control crop constraints such as leaf area index [68], plant height, colour, shape, size of leaves [69] etc. IOT and UAV are also convenient in controlling soil moisture [70-71]; agriculture water constraints like $\mathrm{pH}$ level [72]; and also climate parameters like wind speed, wind direction, rainfall, radiation, air pressure, temperature, relative humidity, , etc. [73-74]. Additionally, remote sensing is also presence very efficiently. Owing to the trivial of sensors, remote sensors are connected in lower altitude UAVs and therefore can control crops efficiently and cost-effectively. Thus, high-resolution records are being achieved by removing different types of unnatural condition like weather condition.

\section{Smart pest management}

Usually, the pest management depends on three features: sensing, evaluating, and treatment. The advanced infection and pest appreciation methods are established on image processing in which raw pictures are developed through the crop zone using UAVs, or remote sensing satellites. Typically, remote sensor protections large extents and, henceforth, provide higher productivity with lower cost. On the other pointer, UAV IOT sensors are accomplished to maintenance more functions in assembling data, like environment sampling, plant health, and pest situations, in each angle through the crop series. For illustration, IoT-based computerised traps can capture, count, and illustrate insect types, additional uploading statistics to the Cloud for comprehensive investigation, which is not possible through remote sensing.

\section{Smart irrigation}

Agricultural UAVs fixed with cameras have the ability to providing wonderful perceptions into exact concerned zones in the field. Using the cameras, the farmers are capable to regulate the areas of low soil moisture, dehydrated crops, and waterlogged zones and in overall have a intellect of the overall health eminence of crops in the farm. Such exact observing were either not likely with traditional farming, inadequately completed or enormously expensive as specialists have to be contracted to convey the task and obtain satisfactory solutions. However, presently UAVs contribute the farmers for provide extra benefit to organize these themselves.

\section{Livestock monitoring (Animal Husbandry)}

Livestock monitoring is nowadays very tough part of agriculture, which engage a very large number of labor efficiency, which cause drawback of cost effectiveness. In this IOT enable $5 \mathrm{~g}$ drone it is possible to take remotely surveillance on livestock monitoring. Unmanned Aerial Vehicle (UAV) as a tool of farming has attracted the interests of an increasing number of researchers. In this paper, we study the problem of deploying a group of UAVs to track and monitor the livestock such as cattle and sheep in a pasture. We assume all targeted 
animals have been fitted with GPS collars, and the mobility of each targeted animal cannot be ignored. We further assume the number of UAVs is sufficient for covering the entire pasture, and we aim to find the optimal UAVs' deployment for minimizing the average UAV- animal distance. We first introduce a procedure of performing sweep coverage by UAVs. By deploying UAVs to achieving sweep coverage for the entire pasture, the initial locations of all targeted animals can be acquired. Then, determine and update the UAVs' deployment by streaming k-means clustering based on the initial locations and received updated locations from the GPS collars.

\section{Forecasting}

Forecasting is the main features of smart agriculture that provide real-time data and historical data to estimate and calculate some important parameters. Scientific demonstrating and machine learning are samples of apparatuses, which are working for forecasting. UAV provides different machine learning models like the regression model for approximating phosphorus amount in the soil [75], forecasting moisture of soil or detection of plant disease [76], Artificial Neural Networks for forecasting temperatures of the filed [77]; etc.

\section{X.8.1. System model}

Internet of Things is the emerging cutting-edge technology, which is dealing with different multidisciplinary Engineering framework for domestic or commercial Automation library. If we consider 3 Gateways as such gateway1, gateway2, gateway3... If we follow OSI model over here network layers are to be more concentrated. Three gateways are enough to optimize the network traffic for user to UAV band and vice versa. After sensing data transmitted through gateway to online cloud server and from the client machine (mobile, tablet, computer) it may be accessed through different portal with real-time readings.

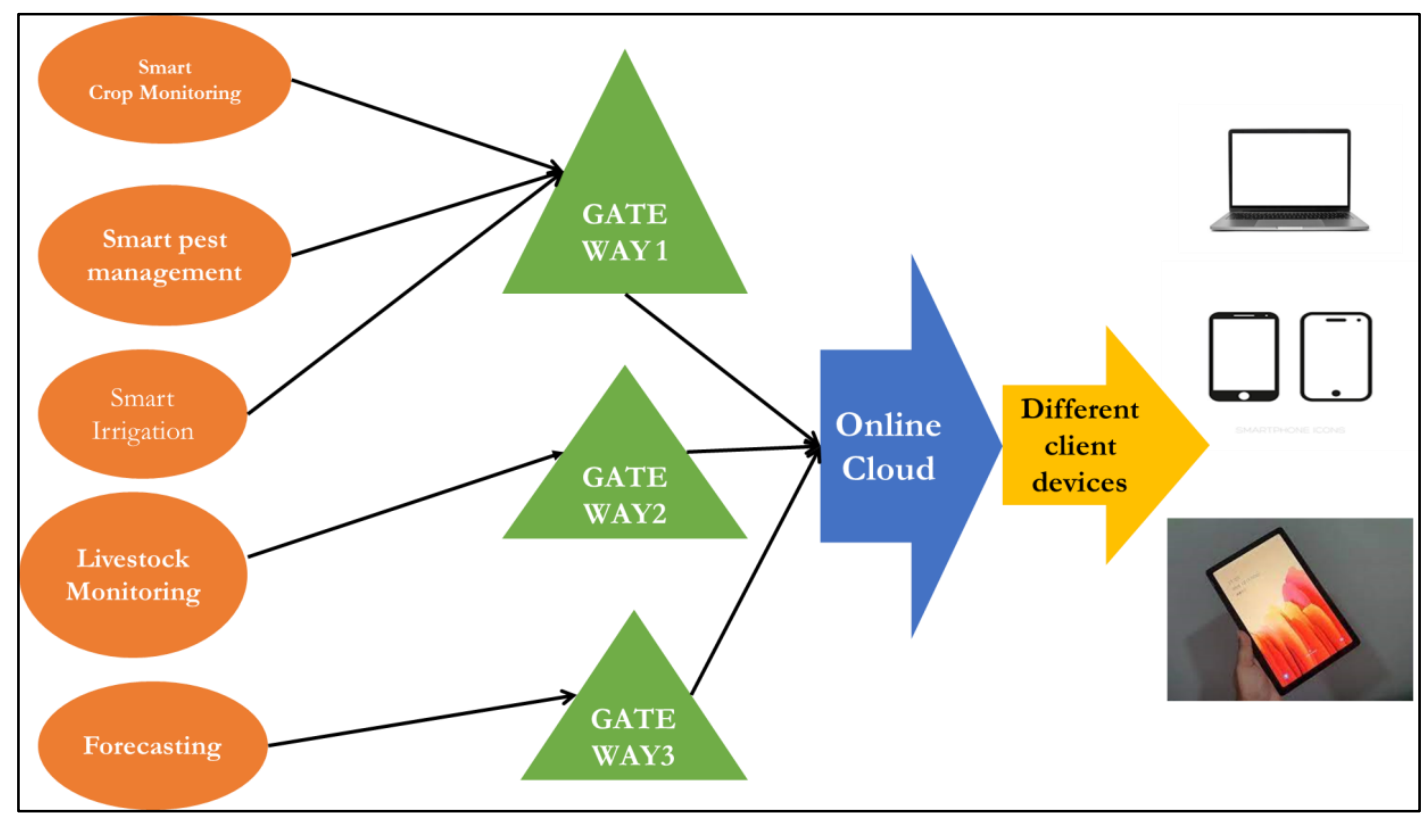

Fig X.5. System model

\section{X.9. Conclusion}

This chapter demonstrate different use cases of smart agriculture, the advantages and application of employing IoT and UAVs in agriculture, the numerous communication technologies and recognizes the problems and restrictions of connectivity of IoT and UAVs in remote areas. The chapter outlined the connectivity restrictions in terms of communication 
technology and transmission range. Moreover, the chapter demonstrate the application of IOT and UAV for smart agriculture through system model.

\section{References}

1. Zavatta G (2014) Agriculture Remains Central to the World Economy, 60\% of the Population Depends on Agriculture for Survival, NetExpo, [Online]

2. Samir, K.C. and Lutz, W., 2017. The human core of the shared socioeconomic pathways: Population scenarios by age, sex and level of education for all countries to 2100. Global Environmental Change, 42, pp.181-192.

3. Le Mouël, C. and Forslund, A., 2017. How can we feed the world in 2050? A review of the responses from global scenario studies. European Review of Agricultural Economics, 44(4), pp.541-591.

4. Chouhan S. S., Singh U. P., and Jain S. (2020) Applications of computer vision in plant pathology: A survey, Arch. Comput. Methods Eng., 27 (2), 611-632

5. Sawe and Benjamin Elisha (2017) WorldAtlas. Countries Most Dependent on Agriculture, Available: https://www.worldatlas.com/articles/countries most-dependent-onagriculture.html

6. FAO in India, Food and Agricultural Organisation of United Nations (2017) Accessed, Available: http://www.fao.org/india/fao-inindia/india-at-a-glance/en/2017

7. Sundmaeker H, Verdouw C., Wolfert S, Prez Freire L (2016), Internet of food and farm 2020, Digitising the Industry - Internet of Things Connecting Physical, Digital and Virtual Worlds, 2, 129-151.

8. Wolfert S, Ge L., Verdouw C., Bogaardt M.-J. (2017), Big data in smart farming a review, Agricultural Systems, 153, $69-80$.

9. Walter A., Finger R., Huber R., Buchmann N., Opinion: Smart farming is key to developing sustainable agriculture, Proceedings of the National Academy of Sciences, 114 (24), 6148-6150

10. Nukala R., Panduru K., Shields A., Riordan D, Doody P., Walsh J. (2016) Internet of things: A review from farm to fork, in: 2016 27th Irish Signals and Systems Conference (ISSC), 1-6.

11. Wong S (2019) Decentralised, Off-Grid Solar Pump Irrigation Systems in Developing Countries-Are They Pro-poor, Pro-environment and Prowomen?, Springer International Publishing, Cham, 367-382.

12. Atzori L, Iera A., Morabito G. (2010) The internet of things: A survey, Computer Networks, 54 (15), 2787 2805

13. Al-Fuqaha A., Guizani M., Mohammadi M, Aledhari M., Ayyash M (2015) Internet of things: A survey on enabling technologies, protocols, and applications, IEEE Communications Surveys Tutorials, 17 (4), 2347-2376

14. Medela, A.; Cendón, B.; González, L.; Crespo, R.; Nevares, I. (2013), IoT multiplatform networking to monitor and control wineries and vineyards, In Proceedings of the 2013 Future Network Mobile Summit, Lisboa, Portugal, 1-10

15. Zheng, R.; Zhang, T.; Liu, Z.; Wang, H. (2016) An EIoT system designed for ecological and environmental management of the Xianghe Segment of China's Grand Canal. Int. J. Sustain. Dev. World Ecol, 23, 372-380.

16. Torres-Ruiz, M.; Juárez-Hipólito, J.H.; Lytras, M.D.; Moreno-Ibarra, M. (2016) Environmental noise sensing approach based on volunteered geographic information and spatio-temporal analysis with machine learning. In Proceedings of the International Conference on Computational Science and Its Applications, Beijing, China, 95-110

17. Hachem, S.; Mallet, V.; Ventura, R.; Pathak, A.; Issarny, V.; Raverdy, P.G.; Bhatia, R. (2015) Monitoring noise pollution using the urban civics middleware, In Proceedings of the 2015 IEEE First International Conference on Big Data Computing Service and Applications, Redwood City, CA, USA, 52-61.

18. Giorgetti, A.; Lucchi, M.; Tavelli, E.; Barla, M.; Gigli, G.; Casagli, N.; Dardari, D. (2016) A robust wireless sensor network for landslide risk analysis: System design, deployment, and field testing. IEEE Sens. J. 16, 6374-6386 
19. Liu, Z.; Huang, J.; Wang, Q.; Wang, Y.; Fu, J. (2013) Real-time barrier lakes monitoring and warning system based on wireless sensor network, In Proceedings of the 2013 Fourth International Conference on Intelligent Control and Information Processing (ICICIP), Beijing, China, 551-554

20. Glaroudis, D.; Iossifides, A.; Chatzimisios, P. (2020), Survey, comparison and research challenges of IoT application protocols for smart farming, Comput. Netw, 168, 107037

21. Osuch, A.; Przygodzi 'nski, P.; Rybacki, P.; Osuch, E.; Kowalik, I.; Piechnik, L.; Przygodzi 'nski, A.; Herkowiak, M. (2020), Analysis of the Effectiveness of Shielded Band Spraying in Weed Control in Field Crops, 10, 475.

22. Panchasara, H.; Samrat, N.; Islam, N. (2021) Greenhouse Gas Emissions Trends and Mitigation Measures in Australian Agriculture Sector-A Review. Agriculture, 11, 85

23. Villa-Henriksen, A.; Edwards, G.T.; Pesonen, L.A.; Green, O.; Sørensen, C.A.G. (2020) Internet of Things in arable farming: Implementation, applications, challenges and potential. Biosyst. Eng. 191, 60-84

24. Kim, J.; Kim, S.; Ju, C.; Son, H.I. (2019) Unmanned aerial vehicles in agriculture: A review of perspective of platform, control, and applications. IEEE Access, 7, 105100-105115

25. Mogili, U.R.; Deepak, B. (2018) Review on application of drone systems in precision agriculture, Procedia Comput. Sci. 133, 502-509

26. Gubbi, J.; Buyya, R.; Marusic, S.; Palaniswami, M. (2013) Internet of Things (IoT): A vision, architectural elements, and future directions, Future Gener. Comput. Syst. 29, 1645-1660.

27. Berni J, Zarco-Tejada P, Suárez L, González-Dugo V, and Fereres E (2009). Remote Sensing of Vegetation from UAV Platforms using Lightweight Multispectral and Thermal Imaging Sensors. Int.Arch.Photogramm.Remote Sen.Spatial Inform Sci, 38(6).

28. Elsenbeiss H, and Sauerbier M (2011). Investigation of uav systems and flight modes for photogrammetric applications. The Photogrammetric Record, 400-421.

29. Thomasson J A, Sui R, and GE Y (2011). Remote sensing of soil properties in precision agriculture:a review. Front Earth Sci, 229-238.

30. Elarab M, Ticlavilca A M, Torres-Rua A F, Maslova I, and McKee M (2015). Estimating chlorophyll with thermal and broadband multispectral high resolution imagery from an unmanned aerial system using relevance vector machines for precision agriculture. International Journal of Applied Earth Observation and Geoinformation, 32-42.

31. Zhang Y, Wang L, and Duan Y (2016). Agricultural information dissemination using ICTs: A review and analysis of information dissemination models in China. Information Processing in Agriculture, 17-29.

32. Cano E, Horton R, Liljegren C, and Bulanon D (2017). Comparison of Small Unmanned Aerial Vehicles Performance Using Image Processing. Journal of Imaging, 1-14.

33. Mukherjee A., Misra S., Sukrutha A., and Raghuwanshi N. S. (2020) Distributed aerial processing for IoTbased edge UAV swarms in smart farming, Comput. Netw., 167

34. Bacco M, Berton A., Gotta A., and Caviglione L. (2018) IEEE 802.15.4 air-ground UAV communications in smart farming scenarios, IEEE Commun. Lett., 22 (9), 1910-1913,

35. Allred B., Martinez L., Fessehazion M. K., Rouse G., Williamson T. N., Wishart D., Koganti T., Freeland R., Eash N., Batschelet A., and Featheringill R. (2020) Overall results and key findings on the use of UAV visible-color, multispectral, and thermal infrared imagery to map agricultural drainage pipes, Agricult. Water Manage., 232, 106036

36. Vroegindeweij BA, van Wijk SW, van Henten E. (2014) Autonomous unmanned aerial vehicles for agricultural applications. In: Proceeding. International Conference of Agricultural Engineering (AgEng), 8

37. Sylvester G (2018). E-Agriculture in Action: Drones for Agriculture. Bangkok: Published by Food and Agriculture Organization of the United Nations and International Telecommunication Union; 
38. Chapman S, Merz T, Chan A, Jackway P, Hrabar S, Dreccer M, et al (2014). Pheno-copter: A low-altitude, autonomous remote-sensing robotic helicopter for high-throughput fieldbased phenotyping. Agronomy, 4(2), 279-301

39. Sugiura R, Noguchi N, Ishii K. (2005) Remote-sensing technology for vegetation monitoring using an unmanned helicopter. Biosystems Engineering, 90(4), 369-379

40. Torres-Sanchez J, Lopez-Granados F, De Castro A, Pena-Barragan J. (2013) Configuration and specifications of an unmanned aerial vehicle (UAV) for early site specific weed management. PLoS One., 8(3), 58210

41. Shafian S, Rajan N, Schnell R, Bagavathiannan M, Valasek J, Shi Y, et al. (2018) Unmanned aerial systemsbased remote sensing for monitoring sorghum growth and development. PLoS One., 13(5), 0196605

42. Zhang J, Basso B, Price RF, Putman G, Shuai G. (2018), Estimating plant distance in maize using unmanned aerial vehicle (UAV), PLoS One., 13 (4), 0195223.

43. Lv M, Xiao S, Tang Y, He Y. (2019) Influence of UAV flight speed on droplet deposition characteristics with the application of infrared thermal imaging, International Journal of Agricultural and Biological Engineering, 12(3), 10-17

44. Ayaz, M.; Ammad-Uddin, M.; Sharif, Z.; Mansour, A.; Aggoune, E.H.M. (2019) Internet-of-Things (IoT)Based Smart Agriculture: Toward Making the Fields Talk, IEEE Access, 7, 129551-129583.

45. Osuch, A.; Przygodzi` nski, P.; Rybacki, P.; Osuch, E.; Kowalik, I.; Piechnik, L.; Przygodzi’ nski, A.; Herkowiak, M. (2020), Analysis of the Effectiveness of Shielded Band Spraying in Weed Control in Field Crops, Agronomy, 10, 475.

46. Villa-Henriksen, A.; Edwards, G.T.; Pesonen, L.A.; Green, O.; Sørensen, C.A.G. (2020) Internet of Things in arable farming: Implementation, applications, challenges and potential. Biosyst. Eng. 191, 60-84

47. Kim, J.; Kim, S.; Ju, C.; Son, H.I. (2019) Unmanned aerial vehicles in agriculture: A review of perspective of platform, control, and applications. IEEE Access, 7, 105100-105115

48. Mogili, U.R.; Deepak, B. (2018) Review on application of drone systems in precision agriculture, Procedia Comput. Sci. 133, 502-509

49. Agarwal, P.; Singh, V.; Saini, G.; Panwar, D. (2019) Sustainable Smart-farming framework: smart farming. In Smart Farming Technologies for Sustainable Agricultural Development; IGI Global: Hershey, PA, USA, $147-173$

50. Bacco, M.; Berton, A.; Ferro, E.; Gennaro, C.; Gotta, A.; Matteoli, S.; Paonessa, F.; Ruggeri, M.; Virone, G.; Zanella, A. (2018) Smart farming: Opportunities, challenges and technology enablers. In Proceedings of the 2018 IoT Vertical and Topical Summit on Agriculture-Tuscany (IOT Tuscany), Tuscany, Italy, 1-6.

51. Hunter, M.C.; Smith, R.G.; Schipanski, M.E.; Atwood, L.W.; Mortensen, D.A. (2017) Agriculture in 2050: Recalibrating targets for sustainable intensification. Bioscience, 67, 386-391.

52. Tzounis, A.; Katsoulas, N.; Bartzanas, T.; Kittas, C. (2017) Internet of Things in agriculture, recent advances and future challenges, Biosyst. Eng., 164, 31-48.

53. Gubbi, J.; Buyya, R.; Marusic, S.; Palaniswami, M. (2013) Internet of Things (IoT): A vision, architectural elements, and future directions, Future Gener. Comput. Syst. 29, 1645-1660.

54. Suhonen, J.; Kohvakka, M.; Kaseva, V.; Hämäläinen, T.D.; Hännikäinen, M. (2012) Low-Power Wireless Sensor Networks: Protocols, Services and Applications; Springer Science \& Business Media: New York, NY, USA,

55. Feng, X.; Yan, F.; Liu, X. (2019) Study of wireless communication technologies on Internet of Things for precision agriculture. Wirel. Pers. Commun. 108, 1785-1802.

56. Jain, R. (2016) Wireless Protocols for IoT Part II: IEEE 802.15.4Wireless Personal Area Networks; IEEE: Saint Louise, MO, USA,

57. Shi, X.; An, X.; Zhao, Q.; Liu, H.; Xia, L.; Sun, X.; Guo, Y. (2019) State-of-the-art internet of things in protected agriculture. Sensor, 19, 1833 
58. Le, N.T.; Hossain, M.A.; Islam, A.; Kim, D.y.; Choi, Y.J.; Jang, Y.M. (2016) Survey of promising technologies for $5 \mathrm{G}$ networks. Mob. Inf. Syst.

59. Beecham Research. (2016). An Introduction to LPWA Public Service Categories: Matching Services to IoT Applications.

60. Atoev, S.; Kwon, K.R.; Lee, S.H.; Moon, K.S. (2017) Data analysis of the MAVLink communication protocol. In Proceedings of the 2017 International Conference on Information Science and Communications Technologies (ICISCT), Tashkent, Uzbekistan, 1-3.

61. Salaan, C.J.; Tadakuma, K.; Okada, Y.; Sakai, Y.; Ohno, K.; Tadokoro, S. (2019) Development and experimental validation of aerial vehicle with passive rotating shell on each rotor. IEEE Robot. Autom. Lett., 4, $2568-2575$.

62. Coombes, M.; Chen, W.H.; Liu, C. (2018) Fixed wing uav survey coverage path planning in wind for improving existing ground control station software, In Proceedings of the 2018 37th Chinese Control Conference (CCC), Wuhan, China, 9820-9825

63. Bhandari, S.; Raheja, A.; Chaichi, M.R.; Green, R.L.; Do, D.; Pham, F.H.; Ansari, M.; Wolf, J.G.; Sherman, T.M.; Espinas, A. (2018) Lessons learned from uav-based remote sensing for precision agriculture. In Proceedings of the 2018 International Conference on Unmanned Aircraft Systems (ICUAS), Dallas, TX, USA, $458-467$.

64. Alsalam, B.H.Y.; Morton, K.; Campbell, D.; Gonzalez, F. (2017) Autonomous UAV with vision based onboard decision making for remote sensing and precision agriculture. In Proceedings of the 2017 IEEE Aerospace Conference, Big Sky, MT, USA, 1-12.

65. Saha, A.K.; Saha, J.; Ray, R.; Sircar, S.; Dutta, S.; Chattopadhyay, S.P.; Saha, H.N. (2018) IOT-based drone for improvement of crop quality in agricultural field, In Proceedings of the 2018 IEEE 8th Annual Computing and CommunicationWorkshop and Conference (CCWC), Las Vegas, NV, USA, 612-615.

66. Muchiri, N.; Kimathi, S. (2016) A review of applications and potential applications of UAV. In Proceedings of the Sustainable Research and Innovation Conference, Rovinj, Croatia, 280-283.

67. M. A. Uddin, A. Mansour, D. Le Jeune, M. Ayaz, and E.-H. Aggoune (2018) UAV-assisted dynamic clustering of wireless sensor networks for crop health monitoring, Sensors, 18(2), 555

68. Orlando, F.; Movedi, E.; Coduto, D.; Parisi, S.; Brancadoro, L.; Pagani, V.; Guarneri, T.; Confalonieri, R. (2016) Estimating leaf area index (LAI) in vineyards using the Pocket LAI smart-app. Sensors, 16, 2004.

69. Dadshani, S.; Kurakin, A.; Amanov, S. (2015) Non-invasive assessment of leaf water status using a dualmode microwave resonator, Plant Methods, 11, 8.

70. Sahota, H.; Kumar, R.; Kamal, A. (2011) A wireless sensor network for precision agriculture and its performance, Wirel. Commun. Mob. Comput, 11, 1628-1645

71. Vellidis, G.; Garrick, V.; Pocknee, S.; Perry, C.; Kvien, C.; Tucker, M. (2007) How wireless will change agriculture. In Proceedings of the Sixth European Conference on Precision Agriculture (6ECPA), Skiathos, Greece, 57-67.

72. Islam, N.; Ray, B.; Pasandideh, F. (2020) IoT Based Smart Farming: Are the LPWAN Technologies Suitable for Remote Communication? In Proceedings of the 2020 IEEE International Conference on Smart Internet of Things (SmartIoT), Beijing, China, 270-276

73. Crabit, A.; Colin, F.; Bailly, J.S.; Ayroles, H.; Garnier, F. (2011) Soft water level sensors for characterizing the hydrological behaviour of agricultural catchments. Sensors, 11, 4656-4673.

74. Navulur, S.; Prasad, M.G. (2017) Agricultural management through wireless sensors and internet of things. Int. J. Electr. Comput. Eng., 7, 3492.

75. Estrada-López, J.J.; Castillo-Atoche, A.A.; Vázquez-Castillo, J.; Sánchez-Sinencio, E. (2018) Smart soil parameters estimation system using an autonomous wireless sensor network with dynamic power management strategy. IEEE Sens. J. 18, 8913-8923 
76. Gao, J.; Nuyttens, D.; Lootens, P.; He, Y.; Pieters, J.G. (2018) Recognising weeds in a maize crop using a random forest machine-learning algorithm and near-infrared snapshot mosaic hyperspectral imagery. Biosyst. Eng., 170, 39-50.

77. Aliev, K.; Pasero, E.; Jawaid, M.M.; Narejo, S.; Pulatov, A. (2018) Internet of plants application for smart agriculture, Int. J. Adv. Comput. Sci. Appl, 9, 421-429. 